\title{
Charge freezing and surface anisotropy on magnetite (100)
}

\author{
J. M. D. Coey and I. V. Shvets \\ Department of Pure and Applied Physics, Trinity College, Dublin 2, Ireland
}

R. Wiesendanger and $\mathrm{H}-\mathrm{J}$. Güntherodt

Institut für Physik, Universität Basel, CH-4056 Basel, Switzerland

Scanning tunneling microscope images of the (100) surface of slightly nonstoichiometric magnetite taken at room temperature show static arrays of pairs of $\mathrm{Fe}^{2+}$ ions with short-range order, and a charge fluctuation time greater than $10^{3} \mathrm{~s}$. The surface appears to be a Wigner glass with electron pairs localized on adjacent ions as the basic unit. The explanation of Wigner localization at room temperature on the surface only is that the spin-polarized minority-spin band derived from $d_{y z}$ orbitals is stabilized and narrowed by the absence of an apicial oxygen from the $B$-site octahedron. This leads to surface anisotropy where the $\mathrm{Fe}^{2+}$ spins are pinned normal to the $\{100\}$ surfaces. Surface anisotropy is expected to outweigh bulk anisotropy in submicron particles.

\section{INTRODUCTION}

Magnetite is the original example of a material where electronic conduction is by charge hopping. The ideal formula of the oxide $\left[\mathrm{Fe}^{3+}\right]\left\{\mathrm{Fe}^{2+}, \mathrm{Fe}^{3+}\right\} \mathrm{O}_{4}$ has an equal mixture of $\mathrm{Fe}^{2+}$ and $\mathrm{Fe}^{3+}$ ions on the octahedral $B$ sites of the cubic spinel lattice (Fig. 1). The ferrous ions have an electronic configuration $3 d^{6}$, which differs from that of the ferric ions by the presence of a single $\downarrow$ electron in addition to the ferric $3 d^{5} \uparrow$ core. At room temperature, the $\downarrow$ electrons hop among all the $B$-site ferric cores with a charge fluctuation time of order $10^{-12} \mathrm{~s},{ }^{1}$ giving magnetite its characteristic black color and nearly metallic conductivity $\left(\rho \sim 10^{-4} \Omega \mathrm{m}\right)$. The conduction electrons occupy a narrow, spin-polarized $d$ band ${ }^{2-4}$ where the effective mass is further enhanced by polaron formation. ${ }^{5}$

On cooling below the Verwey transition temperature $T_{v} \sim 120 \mathrm{~K}$, there is a structural and electronic phase transition, marked by a sharp increase in resistivity and a lowering of the symmetry to monoclinic due to the formation of an ordered array of ferrous ions on $B$ sites. ${ }^{5-8}$ The details of the charge ordering are not entirely clear, but is appears that the low-temperature structure may involve pairs of ferrous ions which alternate with pairs of ferric ions along the [110] $B$-site rows, ${ }^{7,8}$ as originally suggested by Mizoguchi. The spacing of the $B$ sites along the rows is $0.30 \mathrm{~nm}$, and the row spacing is $0.6 \mathrm{~nm}$.

Charge ordering under the influence of the Coulomb interaction was discussed theoretically by Wigner in 1938 . In a solid, the critical factor is the ratio of the interatomic Coulomb interaction $V=e^{2} / 4 \pi \varepsilon_{0} \varepsilon d$ to the bandwidth $W$, where $d$ is the appropriate interatomic spacing and $\varepsilon$ is the dielectric constant. When this ratio $V / W$ is greater than about 3, Wigner crystallization occurs. ${ }^{9}$ The ground state of magnetite is considered to be one where Wigner localization has set in. The Verwey transition may be driven by the entropy of the disordered high-temperature state, as well as screening of the interatomic interaction $V$ by thermally excited electrons which increases the dielectric constant $\varepsilon$.

Here we interpret recently published scanning tunneling microscope images of a clean magnetite surface at room temperature ${ }^{10-12}$ in terms of Verwey-type charge order in the surface layer. Implications concerning surface magnetic anisotropy of ferrites are discussed.

\section{EXPERIMENTAL RESULTS}

A clean, unreconstructed (100) surface of a natural single crystal of magnetite was produced by polishing and annealing in ultrahigh vacuum $\left(10^{-11} \mathrm{mbar}\right)$, as described in Ref. 10. Scanning tunneling microscope images with atomic resolution were first obtained with a normal tungsten tip. Some of the images showed rows of atoms with a spacing of $0.3 \mathrm{~nm}$ between the atoms and a spacing of 0.6 $\mathrm{nm}$ between the rows. Steps of $0.2 \mathrm{~nm}$ are found, with the rows of atoms turned through $90^{\circ} .11,12$ The atoms are identified as the $B$-site iron ions which form rows in the [110] directions. The oxygens are not imaged with the experimental conditions used. ${ }^{11}$ Other planes containing $A$-site iron with clearly different topography were also observed, but they are not relevant to the present discussion.

A remarkable effect was observed when an atomically sharp iron tip ${ }^{10}$ was substituted for the tungsten one. Instead of uniform rows of $B$-site iron atoms, a marked contrast appears along the rows on a scale corresponding to pairs of iron atoms, as shown in Fig. 2. It seems that the iron tip somehow provides magnetic contrast which per-

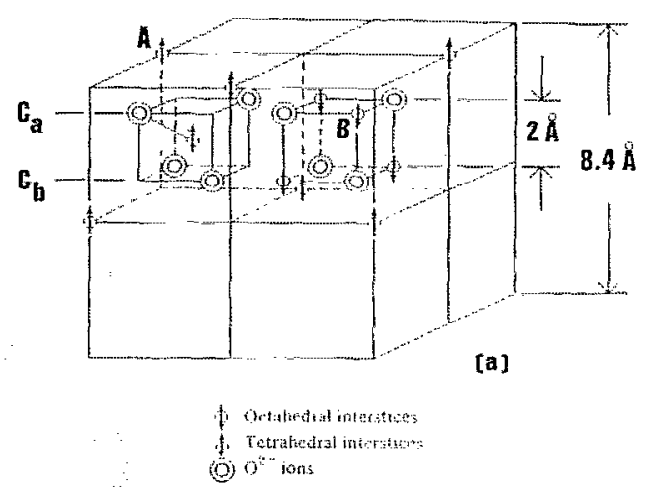

FIG. 1. Crystal structure of magnetite. 


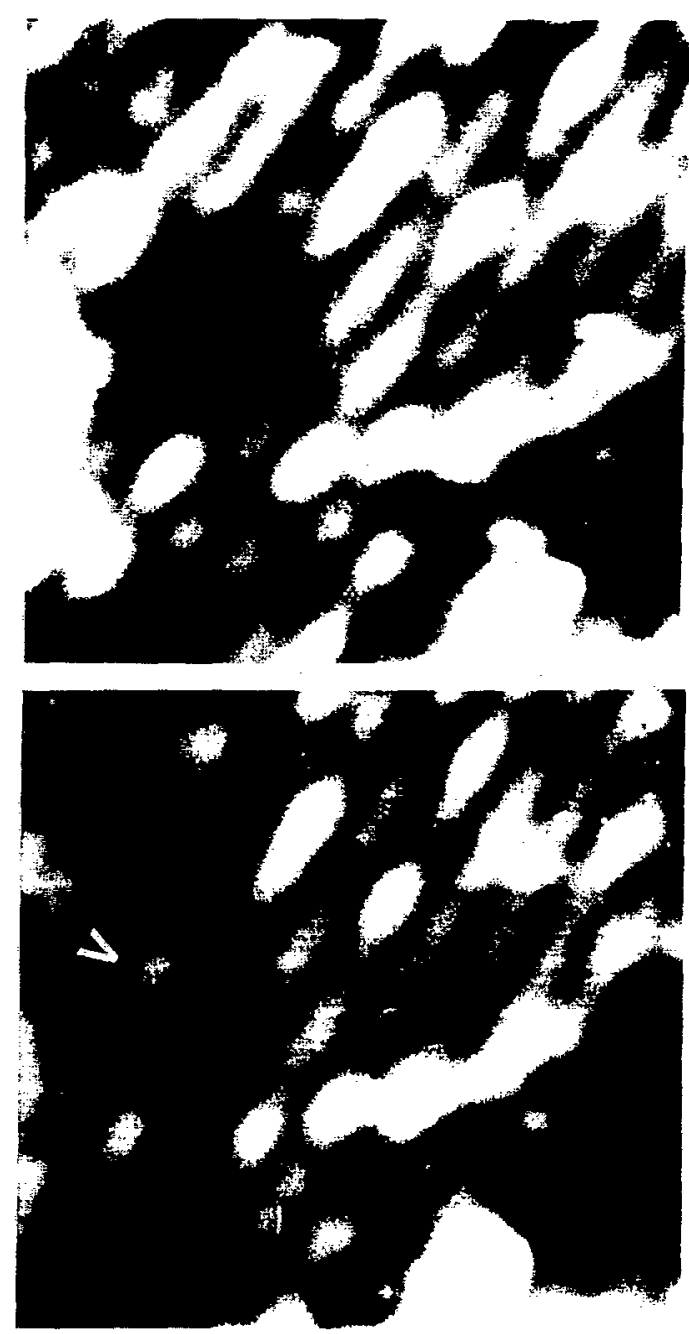

FIG. 2. Scanning tunneling microscope images of the (100) magnetite surface obtained with an iron tip; (b) is a pieture of the same area as (a), taken 10 min later. The total grey scale corresponds to about $1 \AA$ for both images. $(I=1 \mathrm{nA}, U=+3.0 \mathrm{~V}$.)

mits the resolution of pairs of $\mathrm{Fe}^{2+}$ and pairs of $\mathrm{Fe}^{3+}$ ions. A likely physical contrast mechanism is spin-dependent electron tunneling from the iron tip, since the $\mathrm{Fe}^{2+}$ ions have a large local $\downarrow$ density of states near the Fermi level, which is absent for the $\mathrm{Fe}^{3+}$ ions. The tunnel current will then depend on the relative orientation of the surface magnetization and the tip magnetization. ${ }^{13}$ Alternatively, there may be a much enhanced sensitivity of the iron tip to tunneling from $3 d$ states, as compared with the tungsten tip. Whatever the reason, the observation of a static pattern of $\mathrm{Fe}^{2+}$ and $\mathrm{Fe}^{3+}$ ions on the magnetite surface at room temperature was totally unexpected. Occasionally, on rescanning the same area some minutes later, a $B$-site pair was observed to have hopped to a different position, as shown by the arrow in Fig. 2(b). This could be a spontaneous fluctuation, or else it might have been induced by surfaceprobe interaction. In any case, the charge fluctuation time on the surface is greater than $10^{3} \mathrm{~s}$. Figure 3 shows the interpretation of the images in Fig. 2, in terms of a static array of $\mathrm{Fe}^{2+}$ and $\mathrm{Fe}^{3+}$ ions.

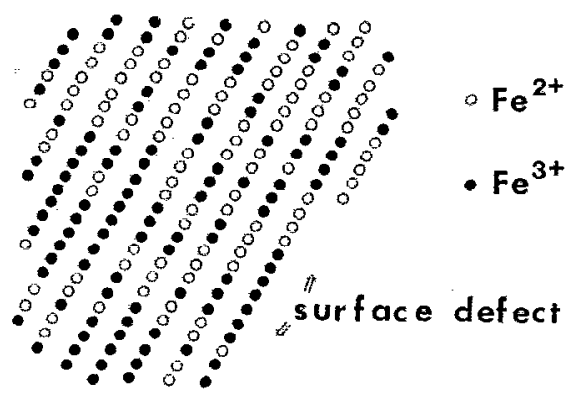

FIG. 3. Interpretation of the images of Fig. 2(b) in terms of an array of $\mathrm{Fe}^{2+}$ and $\mathrm{Fe}^{3+}$ ions.

\section{DISCUSSION}

The experiments indicate that $\mathrm{Fe}^{2+}$ and $\mathrm{Fe}^{3+}$ ions are ordered statically on the (100) surface of magnetite, while there are rapid electronic fluctuations in the bulk. Conversion-electron Mössbauer spectra on a $0.5 \mathrm{~nm}$ (111) surface layer of ${ }^{57} \mathrm{Fe}_{3} \mathrm{O}_{4}$ (Ref. 14) showed that half the Mössbauer area was in a normal room-temperature magnetite pattern, with an averaged $B$-site spectrum due to rapid charge fluctuations. This suggests that only in the topmost layer has the normal electron hopping been frozen out. How can this come about?

The essential difference between the $B$ sites on the surface and in the bulk is the oxygen coordination. The surface sites are missing an apical oxygen from the surrounding octahedron (Fig. 4). Considering only the oxygen neighbors, the site symmetry is lowered from approximately cubic to tetragonal $(4 \mathrm{~mm})$ which leads to an Isingtype $| \pm 1\rangle$ orbital doublet ground state for $\mathrm{Fe}^{2+} \cdot{ }^{16}$ For $\mathrm{Fe}^{2+}$ it is appropriate to consider the one-electron energy levels corresponding to the single $\downarrow$ electron. The $t_{2 g}$ levels are split, as shown in Fig. 5, with the levels corresponding to the $y z$ and $z x$ orbitals lying lower than that of the $x y$ orbital. (The $z$ axis is perpendicular to the surface, and the $x$ axis is along the [110] row.) The easy direction then lies along the $z$ axis normal to the surface. ${ }^{16}$ At the tip, shape anisotropy is expected to favor a parallel direction, which would be the reason for magnetic contrast. The easy directions for magnetite at room temperature are (111). It follows that there is magnetic reconstruction near the surface $^{17}$ in a layer of thickness comparable to the domain wall width $(\sim 500 \AA)$, where the magnetization rotates from the (001) direction to (111).
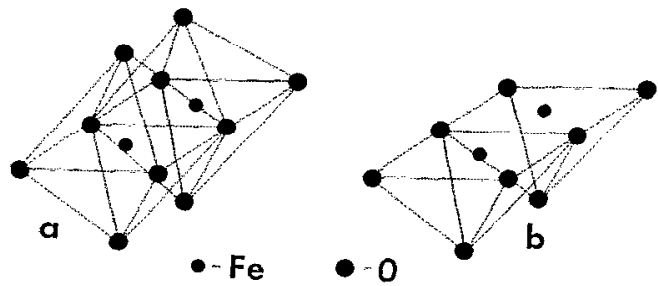

FIG. 4. $B$-site ions in the bulk (a) and on the (100) surface (b). 


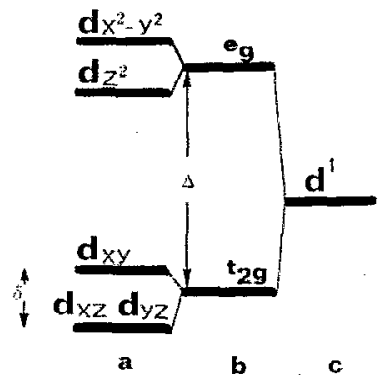

FIG. 5. $B$-site electronic structure at the surface of magnetite. The splitting (a) is for the case when the nearest oxygen neighbors are taken into account and the symmetry of the crystal field is tetragonal; (b) is the splitting in octahedral oxygen corrdination; and (c) is for the zero crystal fiela.

When the next neighbors are taken into account the symmetry becomes orthorhombic, and it is even lower when charge ordering is included. From electrostatic considerations, the subband derived from the $y z$ orbitals is expected to be narrower, and generally lower than the $z x$ subband. There will be minimum overlap of the orbitals of the $\mathrm{Fe}^{2+}$ "triplet pairs," when $y z$ are occupied. In terms of the criterion for Wigner localization, $W$ is drastically reduced at the surface, whereas $V$ may remain essentially unchanged. This conclusion should remain qualitatively unaltered when covalent mixing with $2 p(\mathrm{O})$ (Refs. 4 and 15 ) is taken into account, since there is one less ligand at the surface. Wigner localization and the Verwey transition therefore occur on the surface before they take place in the bulk. Similar results are to be expected in other chargehopping conductors.

The term "Wigner glass" was previously used by Mott ${ }^{5}$ to describe the electronic structure of magnetite above $T_{v}$. We see here that it applies in a fuller sense to the (100) surface, where the electrons are statically frozen in a pattern of short-range order, but not long-range crystallization. As regards the metallic state, both the present data and the charge-ordering schemes below $T_{1}$ (Refs. 7 and 8 ) suggest that pairs of $\mathrm{Fe}^{2+}$ ions are the basic unit. The Coulomb repulsion at short range is apparently overcome by the gain in energy achieved by a pair of ferrous ions sharing the distortion of their surrounding oxygen cages; the interatomic Coulomb repulsion $V$ operates among these basic units. If they persist into the metallic state, as the fluctuation in Fig. 2(b) suggests, then the charge car- riers above $T_{v}$ are bipolarons rather than single polarons. The structure observed on the (100) magnetite surface is a bipolaron glass. Existence of bipolarons above $T_{v}$ in the bulk was experimentally observed in Ref. 18 .

The magnitude of the surface anisotropy associated with the $\mathrm{Fe}^{2}+$ ions in the truncated octahedra may be roughly estimated as $\delta^{2} / \Delta$, where $\delta$ is the splitting of the $t_{2 g}$ levels and $\Delta$ is the overall crystal-field splitting. A typical value would be $10-100 \mathrm{~K} /$ ion, or $\sim 10^{-3} \mathrm{~J} / \mathrm{m}^{2}$. This anisotropy, which pins the magnetization in directions roughly normal to the $\{100\}$ faces of a particle may account for the reduced magnetization in fine particles of magnetite and other spinels containing $\mathrm{Fe}^{2+}$ ions. In fact, the surface anisotropy will be the dominant term in submicron particles. However, this anisotropy should be largely eliminated by any surface treatment which restores the apical oxygen, for example adsorbing molecules of a suitable gas onto the surface.

\section{ACKNOWLEDGMENTS}

Financial support of the Irish Science and Technology Agency EOLAS and the Swiss National Science Foundation is gratefully acknowledged.

T, Mizoguchi and M. Inoue, J. Phys. Soc. Jpn. 21, 1310 (1966).

${ }^{2}$ D. L. Camphausen, J. M. D. Coey, and B. K. Chakraverty, Phys. Rew. Lett. 29, 657 (1972)

${ }^{3}$ S. F. Alvarado, M. Erbudak, and P. Munz, Phys. Rev. B 14, 2740 (1976).

${ }^{4}$ A. Yanase and K. Siratori, J. Phys. Soc. Jpn. 53, 312 (1984).

${ }^{5}$ N. F. Mott, Metal Insulator Transitions, 2nd ed. (Taylor \& Francis, London, 1990), Chap. 8.

${ }^{6}$ R. Aragon, D. J. Buttery, J. P. Shepherd, and J. M. Honig, Phys. Rev. B 31, 430 (1985).

'S. IIda, K. Mizushima, M. Mizoguchi, K. Kose, K. Kato, K. Yanaí, N. Goto, and S. Yumoto, J. Appl. Phys. 53, 2164 (1982).

${ }^{8}$ E. Kita, Y. Tokuyama, A. Tasaki, and K. Siratori, J. Magn. Magn. Mater. 31-34, 787 (1983).

${ }^{9}$ J. R. Cullen and E. Callen, Phys. Rev. B 7, 397 (1973).

${ }^{10}$ I. Shvets, R. Wiesendanger, D. Bürgler, G. Tarrach, H-J. Güntherodt, and J. M. D. Coey, J. Appl. Phys. 71, 5489 (1992).

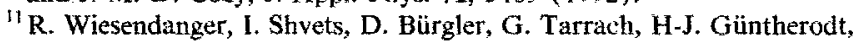
J. M. D. Coey, and S. Gräser, Science 255, 583 (1992).

${ }^{12} \mathrm{R}$. Wiesendanger, I. Shvets, D. Bürgler, G. Tarrach H-J, Güntherodt, and J. M. D. Coey, Europhys. Lett. 19, 141 (1992).

13. J. C. Slonczewski, Phys. Rev. B 39, 6995 (1988).

${ }^{14}$ T. Fujii, M. Takano, R. Katano, Y. Bando, and Y. Isozumi, J. Appl. Phys. 68, 1735 (1990).

${ }^{15}$ M. Sancrotti, F. Ciccacei, M. Finazzi, E. Vescove, and S. F. Alvarado, Z. Phys. B 84, 243 (1991).

${ }^{16}$ F. Varret, J. Phys. 37, C6-437 (1976).

${ }^{17}$ J, R. Cullen, K. B. Hathaway, and J. M. D. Coey, J. Appl. Phys. 63, 3649 (1988).

${ }^{18}$ Y. Yamada, Philos. Mag. B 42, 377 (1980). 